\title{
Localization in Highly Anisotropic Systems
}

\author{
I. Zambetaki, ${ }^{1}$ Qiming Li, ${ }^{2}$ E. N. Economou, ${ }^{1}$ and C. M. Soukoulis, ${ }^{1,2}$ \\ ${ }^{1}$ Research Center of Crete and Department of Physics \\ P. O. Box 1527, 71110 Heraklion, Crete, Greece \\ ${ }^{2}$ Ames Laboratory and Department of Physics and Astronomy, \\ Iowa State University, Ames, IA 50011
}

\begin{abstract}
The localization behavior of the Anderson model with anisotropic hopping integral t for weakly coupled planes and weakly coupled chains is investigated both numerically with the transfer matrix method and analytically within the self-consistent theory of localization. It is found that the mobility edge is independent of the propagating direction. However, the correlation (localization) length in the extended (localized) side of the transition can be very different for the two directions. The critical disorder $\mathrm{W}_{c}$ is found to vary from $\mathrm{t}^{\frac{1}{4}}$ for weakly coupled planes to $\mathrm{t}^{\frac{1}{2}}$ for weakly coupled chains.

PACS numbers: 72.15.Rn 71.30.+h 74.25.Fy
\end{abstract}

The problem of Anderson localization in anisotropic systems has attracted considerable attentiont 15 in recent years, largely due to the fact that the high $\mathrm{T}_{c}$ superconductors are highly anisotropic. Transport in the normal state is metallic in the ab-plane but appears semiconductor-like in the c-axis 6 . The nature of the caxis transport in high $\mathrm{T}_{c}$ materials is still controversial and its understanding may have important consequences for the theories of the normal and the superconducting state This paradoxical property has prompted the proposall that a high $\mathrm{T}_{c}$ material in the normal state is actually an insulator, appearing metallic only because the inelastic length in the plane is less than the localization length. It has also been argued2 1 that a negative $\mathrm{d} \rho / \mathrm{dT}$ in the $\mathrm{c}$ direction alone may signify anisotropic localization, with a metal-insulator transition depending on the propagating direction, in direct contradiction to the predictions of the scaling theory of localizations. A recent diagrammatic calculationl lent support to such a claim. Previous diagrammatic analysis 2 , however, led to the conclusion that the scaling property in anisotropic systems remains the same as that of the isotropic systems with a simple substitution of the conductance by its geometric mean. Given the perturbative nature of all the previous work, it is important to carefully study the localization behavior of disordered anisotropic systems with reliable numerical techniques and to determine whether the scaling theory is valid for highly anisotropic systems 810 .

In the present work, we have systematically studied the localization properties of a three-dimensional disordered anisotropic system described by a tight-binding Hamiltonian with random on-site energies and anisotropic hopping integrals. We calculate numerically both the conductance and the localization length of such systems with the transfer-matrix method 0 . By doing finite-size scaling analysis on sufficiently large systems, we find the metal-insulator transition occurs at the same critical disorder $W_{c}$ in all directions. Remarkably, at criticality, the geometric mean of the ratio of the finite-size localization length to the width of the bar is independent of the anisotropy. This underlies the conformal invariance property at the Anderson transition. For systems with extremely weak plane-coupling, the critical disorder $W_{c}$ is found to vary with anisotropy $t$ as $t^{\frac{1}{4}}$, in good agreement with a self-consistent theory of localization. However, the correlation lengths in the extended regime, as well as the localization lengths in the localized regime, differ tremendously in different directions. As we will discuss later, this difference of the correlation lengths in the propagating directions may explain the c-axis transport of the high- $\mathrm{T}_{c}$ materials. The localization behavior of weakly coupled disordered chains, which simulate the 1D-to-3D behavior, is also studied. The critical disorder $\mathrm{W}_{c}$ is found to behave as $\mathrm{t}^{\frac{1}{2}}$.

We study the following Hamiltonian for an anisotropic 3D disordered model

$$
H=\sum_{n} \epsilon_{n}|n><n|+\sum_{n, m} t_{n m}|n><m|
$$

where the sites $\{n\}$ form a regular cubic lattice. The hopping integrals are nonzero between nearest-neighbor sites only and depend on directions, in general, $t_{x} \neq t_{y} \neq t_{z}$. We normalize all energies by the largest hopping integral. For systems with weak plane coupling, the hopping integrals are given by $\{1,1, t\}$. As a convention, we have assigned the direction with the large and small hopping integral as the parallel $(\|)$ and the perpendicular $(\perp)$ direction, respectively. Disorder is introduced by choosing randomly site energies $\epsilon_{n}$ within [-W/2, W/2].

The metal or insulating nature of the Anderson Hamiltonian, Eq. (1), can be determined by investigating the scaling properties of either the conductance or the localization length of finite gystems calculated from the transfer-matrix techniques 11 . The conductance $\mathrm{G}$ of an $\mathrm{M} \times \mathrm{M} \times \mathrm{M}$ cubeis calculated from the multi-channel Landauer formula 12 


$$
G(M)=\frac{e^{2}}{h} \operatorname{Tr}\left(T^{+} T\right)
$$

where $\mathrm{T}$ is the transmission matrix. For the localization length calculation, one considers a bar of length $\mathrm{N}$ and cross-section $\mathrm{M} \times \mathrm{M}$. One determines the largest localization length $\lambda_{M}$ as $\mathrm{N} \rightarrow \infty$ from the smallest Lyapunov coefficient of the product of the random transfer matrices. From a plot of $\lambda_{M} / \mathrm{M}$ (or $\mathrm{G}$ ) vs. M, one can determine the localization properties of the system. For localized states $\left(\mathrm{W}>\mathrm{W}_{c}\right)$ the ratio $\lambda_{M} / \mathrm{M}($ or $\mathrm{G}$ ) is expected to fall with increasing $M$, while for extended states $(\mathrm{W}<$ $\mathrm{W}_{c}$ ) $\lambda_{M} / \mathrm{M}$ (or $\mathrm{G}$ ) should rise instead. At the mobility edge trajectory $\left(\mathrm{W}=\mathrm{W}_{c}\right), \lambda_{M} / \mathrm{M}$ (or $\mathrm{G}$ ) is independent of $\mathrm{M}$ and this behavior defines the Anderson transition point and the critical disorder $\mathrm{W}=\mathrm{W}_{c}$. In our calculations, we have used systems with $\mathrm{M}=5-17$ and $\mathrm{N}$ was at least 5000. For the conductance calculations, M was up to 22 . Due to the nonself-averaging nature of finite-size systems, an average over many random configurations (up to 500 for the $\mathrm{M}=20$ case) must be taken to suppress the large fluctuations.

In Fig. 1, we present our numerical results for the dimensionless conductance $g=\frac{G}{e^{2} / h}$ and the scaled localization length $\lambda_{M} / \mathrm{M}$ versus $\mathrm{M}$ at $\mathrm{E}=0$ for the case of weakly coupled planes with coupling constant $\mathrm{t}=0.1$, for both the perpendicular (lower panels) and parallel (upper panels) directions. These results strongly suggest that for sufficiently large $\mathrm{M}$, the critical disorder defined by an M-independent $\mathrm{g}$ and $\lambda_{M} / \mathrm{M}$ converges to the same value, $\mathrm{W}_{c} \simeq 8.4$, for both propagating directions. Notice that if only sizes $\mathrm{M} \leq 11$ were used, which are appropriate in the isotropic case, one would then have erroneously concluded that $\mathrm{W}_{c}^{\|}>\mathrm{W}_{c}^{\perp}$. Another important point is that the value of $\lambda_{M} / \mathrm{M}$ at $\mathrm{W}=\mathrm{W}_{c}$, called $\Lambda_{c}$, is different in the two propagating directions. We find that $\Lambda_{c}^{\perp}$ $=\mathrm{t} \Lambda_{c}^{\|}$for all the t's we have examined. The critical conductance in the two directions at $\mathrm{t}=0.1$ is approximately $\mathrm{g}_{c}^{\perp} \simeq 10^{-6} \mathrm{~g}_{c} \|$. This is a much stronger variation than the $\mathrm{g}^{\perp} \simeq \mathrm{t}^{2} \mathrm{~g} \|$ predicted by the diagrammatic analysis $\mathrm{I}^{\|}$of the anisotropic model. This may be an indication of the existence of high order corrections to the conductivity that is not simply proportional to the bare conductivity. This point needs further study.

In order to extrapolate to infinite system size $(M \rightarrow \infty)$, it is necessary to investigate the scaling behavior of $\lambda_{M} / \mathrm{M}$. In the center of the band $(\mathrm{E}=0)$ and close enough to the transition point, it turned out to be possible to establish a scaling function for both propagation directions within the accuracy of our numerical results. The scaling function $\mathrm{f}(\mathrm{x})$ behaves as $1 / \mathrm{x}$ in the $\mathrm{x} \rightarrow 0$ limit for extended states (while for localized states $\mathrm{f}(\mathrm{x})$ $\sim \mathrm{x})$. For $\mathrm{x} \rightarrow \infty, \mathrm{f}(\mathrm{x})$ approaches a constant value that depends on the propagating direction. For $\mathrm{t}=0.1$, we obtained $\Lambda_{c}^{\|}=1.2$ and $\Lambda_{c}^{\perp}=0.12$. This suggests that $\Lambda_{c}^{\perp}=\mathrm{t} \Lambda_{c}^{\|}$for general t. We have, indeed, checked that this formula is correct for any t. Another important rela- tion we were able to obtain is the geometric mean of the different critical values $\lambda_{M} / \mathrm{M}$ in the different directions is independent of the anisotropy t. We derived

$$
\left(\Lambda_{c}^{x} \Lambda_{c}^{y} \Lambda_{c}^{z}\right)^{1 / 3}=0.6
$$

In the case of weakly coupled planes, Eq. (3) becomes $\left(\left(\Lambda_{c}^{\|}\right)^{2} \Lambda_{c}^{\perp}\right)^{1 / 3} \simeq 0.6$. This relation may have important consequences for the existence of conformal invariance at the critical point of the Anderson localization problemen, 4 .

In Fig. 2, we plot the localization length $\mathrm{L}_{c}$ and the correlation length $\xi$ as a function of $\mathrm{W}$ for $\mathrm{E}=0$ and $\mathrm{t}$ $=0.1$ for the parallel and the perpendicular directions. Both $\mathrm{L}_{c}$ and $\xi$ diverge at the critical disorder $\mathrm{W}_{c}$. However, both $\xi$ and $\mathrm{L}_{c}$ differ substantially for the different propagating directions. In particular, in the extended regime where $\mathrm{W}<\mathrm{W}_{c}, \xi^{\perp}>\xi^{\|}$. For example, at $\mathrm{W}=5$, $\xi^{\perp}=100$, and $\xi^{\|} \simeq 1.5$, in units of the lattice constant, which has been taken to be one. In the localized regime where $\mathrm{W}>\mathrm{W}_{c}, \mathrm{~L}_{c}^{\|}>\mathrm{L}_{c}^{\perp}$. For example at $\mathrm{W}=10, \mathrm{~L}_{c}^{\|}=55$, and $\mathrm{L}_{c}^{\perp} \simeq 5$. Our numerical results approximately follow the theoretical prediction $\xi^{\|}=t^{2} \xi^{\perp}$ and $L_{c}^{\perp}=t L_{c}^{\|}$. However, the exponents are expected to be the same and this has been confirmed from our calculations. We find that $\nu^{\perp}=1.3 \pm 0.1$ and $\nu^{\|}=1.3 \pm 0.3$, in agreement with each other and with $\nu=1.3 \pm 0.1$ for the isotropic system within the numerical accuracy.

The difference between $\xi^{\|}$and $\xi^{\perp}$ is very important and can possibly explain the normal state transport properties 6 of the high- $\mathrm{T}_{c}$ materials. The correlation length $\xi$ measures the strength of the fluctuations of the wave functions in the extended regime. For length scales larger than $\xi$, the wave function looks uniform, while for length scales smaller than $\xi$ the wave function has strong fluctuations. The relevant length scale is the inelastic mean free path $\ell_{i n}$ which behaves as $\mathrm{T}^{-p}$, with probably $\mathrm{p}=1 / 2$. When $\ell_{i n}<\xi$, a phenomenon called incipient localization takes place and conductivity is controlled by $\ell_{i n}$. A convenient interpolation formula15, valid in the conducting regime is given by

$$
\sigma_{l o c}=\frac{e^{2}}{\hbar}\left(\frac{a}{\xi}+\frac{b}{\ell_{i n}}\right)
$$

where $\mathrm{a}$ and $\mathrm{b}$ are constants of order unity 6 . For high enough temperatures the conductivity is given by the regular metallic behavior, where $\sigma_{p h}=\omega_{p}^{2} \tau / 4 \pi$ and the mean free time $\tau \sim T^{-1}$. Because $\sigma_{p h}$ and $\sigma_{l o c}$ describe independent physical processes, we can add the corresponding resistivities. Therefore $\rho=\rho_{l o c}+\rho_{p h}$, where $\rho=1 / \sigma$. The experimental behavior of the high- $\mathrm{T}_{c}$ materials can be understood if we assume that the high$\mathrm{T}_{c}$ oxides, instead of being insulators anisotropic metals with a large anisotropic correlation length. At low $\mathrm{T}$, the resistivity is dominated by $\rho_{\text {loc }}$. Once $\ell_{i n}$ becomes shorter than $\xi$ in the perpendicular direction (c-direction), $\xi^{\perp}$, Eq. (4) suggests that there is 
an sharp downturn in the perpendicular resistivity as the temperature increases. This trend will eventually stop at some $\mathrm{T}$ when the regular resistivity begin to dominate. Then $\rho^{\perp}$ will start increasing linearly with $\mathrm{T}$, as in the regular metallic behavior. In the parallel direction, $\xi^{\|}$is always smaller than the inelastic length and the transport in the plane remains metallic. The above analysis neglects correlations and dynamic disorder that could also affect substantially the transport properties?.

The dependence of $W_{c}$ on the anisotropy t for the weak plane coupling has been also numerically calculated by the transfer matrix technique. The numerical values of $W_{c}$ can be fitted well with a single power law dependence $\mathrm{W}_{c}=15.4 \mathrm{t}^{\frac{1}{4}}$, for $t<0.8$. This is clearly seen in Fig. 3 . This dependence is in marked contrast to what one would expect based on reasonable heuristic argument 17 and also predicted previously by more elaborate theories 14 which give a much weaker $\mathrm{t}$ dependence, $\mathrm{W}_{c} \sim 1 / \sqrt{|\ell n t|}$. The $\mathrm{t}$ dependence is also different from the results obtained for the weakly coupled chainstes, which give $\mathrm{W}_{c} \sim t^{\frac{1}{2}}$. To understand the $t^{\frac{1}{4}}$ dependence of $\mathrm{W}_{c}$, we examine the localization transition starting from the results of the diagrammatic analysis 9 The maximally crossed diagrams produced a correctiont to the zero-temperature configurationally average conductivity $\sigma_{i o}(\mathrm{i}=\mathrm{x}, \mathrm{y}, \mathrm{z})$ of the form given in Eq. (2.3a) of Ref. 4. An equivalent criterion in the tight-binding representation for the mobility edge is given by Eq. (2.5) of Ref. 4. For the anisotropic case we made the choice that the effective lattice constant $a_{i}$ is proportional to anisotropic mean free path $\ell_{i}$. $\left.\ell_{i} \sim\left(<v_{i}^{2}>\right)^{1 / 2} \tau=\left[\langle v\rangle<\frac{v_{i}^{2}}{v}\right\rangle\right]^{1 / 2} \tau$, i.e., we assume that $\tau$ is isotropic. Since $\sigma_{i o} / a_{i}^{2}$ is independent of $\mathrm{i}$, the localization criterion is satisfied simultaneously in all the directions and can be written as

$$
\frac{\pi \hbar \Omega \sigma_{i o}}{2 e^{2} a_{i}^{2}}=\frac{1}{(2 \pi)^{3}} \int d \vec{q} \frac{1}{\sum_{i=1}^{3}\left(2-2 \cos q_{i}\right)}=G_{3 D}^{i s .}(E=6),
$$

where $\Omega=\prod_{i=1}^{3} a_{i}$ and $2 \mathrm{G}_{3 D}^{i s .}(\mathrm{E}=6)=0.505462$ is the 3D Green's function of the isotropic system at the band edge 19 . Notice the same equation applies to the isotropic case except now both the conductivity and the mean free path enter as geometric means.

In the limit of weak coupling $t \rightarrow 0$, Eq. (5) yields $\tau \sim t^{-\frac{1}{2}}$ for weakly squpled planes and $\tau \sim t^{-1}$ for weakly coupled chainsen, and therefore $\mathrm{W}_{c}$ ( taking into account that $\tau \sim \mathrm{W}^{-2}$ ) is proportional to $\mathrm{t}^{\frac{1}{4}}$ and $\mathrm{t}^{\frac{1}{2}}$, respectively, in agreement with the transfer-matrix method results. We have systematically calculated $20 \mathrm{~W}_{c}$ vs $t$, for general t, within the CPA, by using Eq. (5). There is quantitative agreement between the CPA and the transfer matrix results. We feel the choice of the effective lattice constant $\mathrm{a}_{i}$ being proportional to an anisotropic mean free path $\ell_{i}$ is the proper one and the good agreement with our numerical results further supports this.

In summary, we have numerically studied, by the transfer-matrix method technique, the localization properties of weakly coupled planes. We found that only one mobility edge exists for both propagation directions, i.e., the states in the planar direction become localized with exactly the same amount of disorder as the states in the perpendicular direction. However, the correlation length $\xi$ in the extended side of the transition, as well as the localization length in the localized side, can be very different for the two propagating directions. This behavior of $\xi$ can possibly explain the transport properties of high$\mathrm{T}_{c}$ materials. The critical value of disorder $\mathrm{W}_{c}$ is proportional to $t^{\frac{1}{4}}$ for weakly coupled planes and is proportional to $t^{\frac{1}{2}}$ for weakly coupled chains. These results are found to be in satisfactory agreement with the predictions of the self-consistent theory of localization which incorporates the idea of length scale rescaling. However, the conductance in different directions does not satisfy the relation predicted by the diagrammatic analysis $\mathrm{l}$. Work to further understand the scaling properties of the conductance are in progress.

\section{ACKNOWLEDGMENTS}

We are grateful to A. G. Rojo for sending us his numerical data at the early stage of the project. Ames Laboratory is operated for the U. S. Department of Energy by Iowa State University under contract No. W7405-ENG-82. This work was supported by the Director of Energy Research, Office of Basic Energy Sciences and NATO Grant No. CRG 940647. It was also supported by EU Grants.

${ }^{1}$ A. A. Abrikosov, Phys. Rev. B 50, 1415 (1994).

${ }^{2}$ A. G. Rojo and K. Levin, Phys. Rev. B 48, 16861 (1993) and references therein.

${ }^{3}$ Y. Zha, S. L. Cooper, and D. Pines, unpublished.

${ }^{4}$ Qiming Li, C. M. Soukoulis, E. N. Economou, and G. S. Grest, Phys. Rev. B 40, 2825 (1989).

${ }^{5}$ W. Xue, P. Sheng, Q. J. Chu, and Z. Q. Zhang, Phys. Rev. Lett. 63, 2837 (1989); Phys. Rev. B 42, 4613 (1990); and Q. J. Chu and Z. Q. Zhang, Phys. Rev. B 48, 10761 (1993).

${ }^{6}$ For a review of experiments see Y. Iye, in Physical Properties of High $\mathrm{T}_{c}$ superconductors, Ed. by D. M. Ginzberg (World Scientific, Singapore, 1992).

${ }^{7}$ G. Kotliar et al., Europhys. Lett. 15, 655 (1991).

${ }^{8}$ For a recent review, see B. Kramer and A. MacKinnon, Rep. Progr. Phys. 56, 1469 (1993).

${ }^{9}$ P. Wölfle and R. N. Bhatt, Phys. Rev. B 30, 3542 (1984); R. N. Bhatt, P. Wölfle, and T. V. Ramakrishnan, Phys. Rev. B 32, 569 (1985); and D. Vollhardt and P. Wölfle, Chapter 1 in "Electronic Phase Transitions", Ed. by W. Hanke and Yu. V. Kopaev (Elsevier, 1992), p. 38. 
${ }^{10}$ W. Apel and T. M. Rice, J. Phys. C 16, L1151 (1983).

${ }^{11}$ For a discussion of the two methods, see J.-L Pichard, N. Zanon, Y. Imry, and A. D. Stone, J. de. Phys. (France) 51, 587 (1990).

12 E.N. Economou and C. M. Soukoulis, Phys. Rev. Lett. 46, 618 (1981); D. S. Fisher and P. A. Lee, Phys. Rev. B23, 685 (1981).

13 J.-L. Pichard and G. Sarma, J. Phys. C:14, L617 (1981); J. T. Chalker and P. D. Coddington, ibid, 21, 2665 (1988); and M. Henkel, J. Phys. A 20, L769 (1987).

${ }^{14}$ Qiming Li and C. M. Soukoulis, unpublished.

${ }^{15}$ Y. Imry, Phys. Rev. Lett. 44, 469 (1980); J. Appl. Phys. 52, 1817 (1981); and S. M. Girvin and M. Jonson, Phys. Rev. Lett. 43, 1447 (1979) and Phys. Rev. B 22, 3583 (1980).

${ }^{16}$ Eq. (4) is strictly valid in the weak disorder limit. This can be seen by expanding $\mathrm{g} \sim 1 /\left(\exp \left(2 \mathrm{M} / \lambda_{M}\right)-1\right)$ for weak disorder, where $\lambda_{M}>>M$. So $g \sim \lambda_{M} / M$ and by using the data in Fig. 1(c-d), we obtain $\mathrm{g} \sim \mathrm{b}+\mathrm{aM} / \xi$, and therefore $\sigma \sim \mathrm{b} / \mathrm{M}+\mathrm{a} / \xi$. The coefficient $\mathrm{b}$ is approximately a constant of order $\Lambda_{c}$ away from the critical regime. Notice that $\Lambda_{c}^{\|}=1.2$ and $\Lambda_{c}^{\perp}=0.12$ for $\mathrm{t}=0.1$.

17 To determine the critical disorder $\mathrm{W}_{c}$ where the system crosses over from 1D-to-3D for the weakly coupled chains and from 2D-to-3D for the weakly coupled planes we consider a particle in a particular chain or plane, respectively. With probability $\frac{1}{2(3-d)}$ the particle will hop to another chain or plane after a time and therefore distance proportional to $1 / \mathrm{t}$, where $\mathrm{t}$ is the hopping integral. The localization length $\mathrm{L}_{c}$ in the $1 \mathrm{D}(2 \mathrm{D})$ case is proportional to $\frac{1}{W^{2}}\left(\exp \left(\frac{\alpha}{W^{2}}\right)\right)$, respectively. So the condition $\mathrm{L}_{c} \simeq 1 / \mathrm{t}$, determines the critical disorder $\mathrm{W}_{c}$. For the $1 \mathrm{D}$-to- $3 \mathrm{D}$, we have $1 / \mathrm{W}_{c}^{2} \simeq 1 / t$, and therefore $\mathrm{W}_{c} \sim t^{\frac{1}{2}}$. For the $2 \mathrm{D}$-to$3 \mathrm{D}$ we have $\exp \left(\frac{\alpha}{W_{c}^{2}}\right) \simeq 1 / t$ and therefore $\mathrm{W}_{c} \sim 1 / \sqrt{|\ell n t|}$.

18 N. A. Panagiotidis, S. N. Evangelou, and G. Theodorou, Phys. Rev. B 49, 14122 (1994); O. N. Dorokhor, Solid State Comm. 46, 605 (1983); ibid 51, 381 (1984).

19 E. N. Economou, "Green's Functions in Quantum Physics," Second Edition (Springer, Heidelberg, 1983).

${ }^{20}$ I. Zambetaki, Qiming Li, E. N. Economou, and C. M. Soukoulis, unpublished.

FIG. 1. The conductance $\mathrm{g}\left(\mathrm{a}\right.$ and b) and $\lambda_{M} / \mathrm{M}$ (c and d) plotted as a function of $\mathrm{M}$ for $\mathrm{E}=0, \mathrm{t}=0.1$, and various values of disorder $\mathrm{W}$, for both propagating directions. The mobility edge is at $W_{c} \simeq 8.4$ for both propagating directions.

FIG. 2. Localization length $\mathrm{L}_{c}$ or correlation length $\xi$, obtained by finite size scaling, as a function of disorder $\mathrm{W}$ for $\mathrm{E}=0$ and $\mathrm{t}=0.1$, for the parallel and perpendicular propagating directions. Notice that for $\mathrm{W}<\mathrm{W}_{c} \simeq 8.4, \xi^{\perp}>\xi^{\|}$, while for $\mathrm{W}>\mathrm{W}_{c}, \mathrm{~L}_{c}^{\|}>\mathrm{L}_{c}^{\perp}$.

FIG. 3. The critical disorder $\mathrm{W}_{c}$ for obtaining localized states at $\mathrm{E}=0$ vs the anisotropy $\mathrm{t}$. 

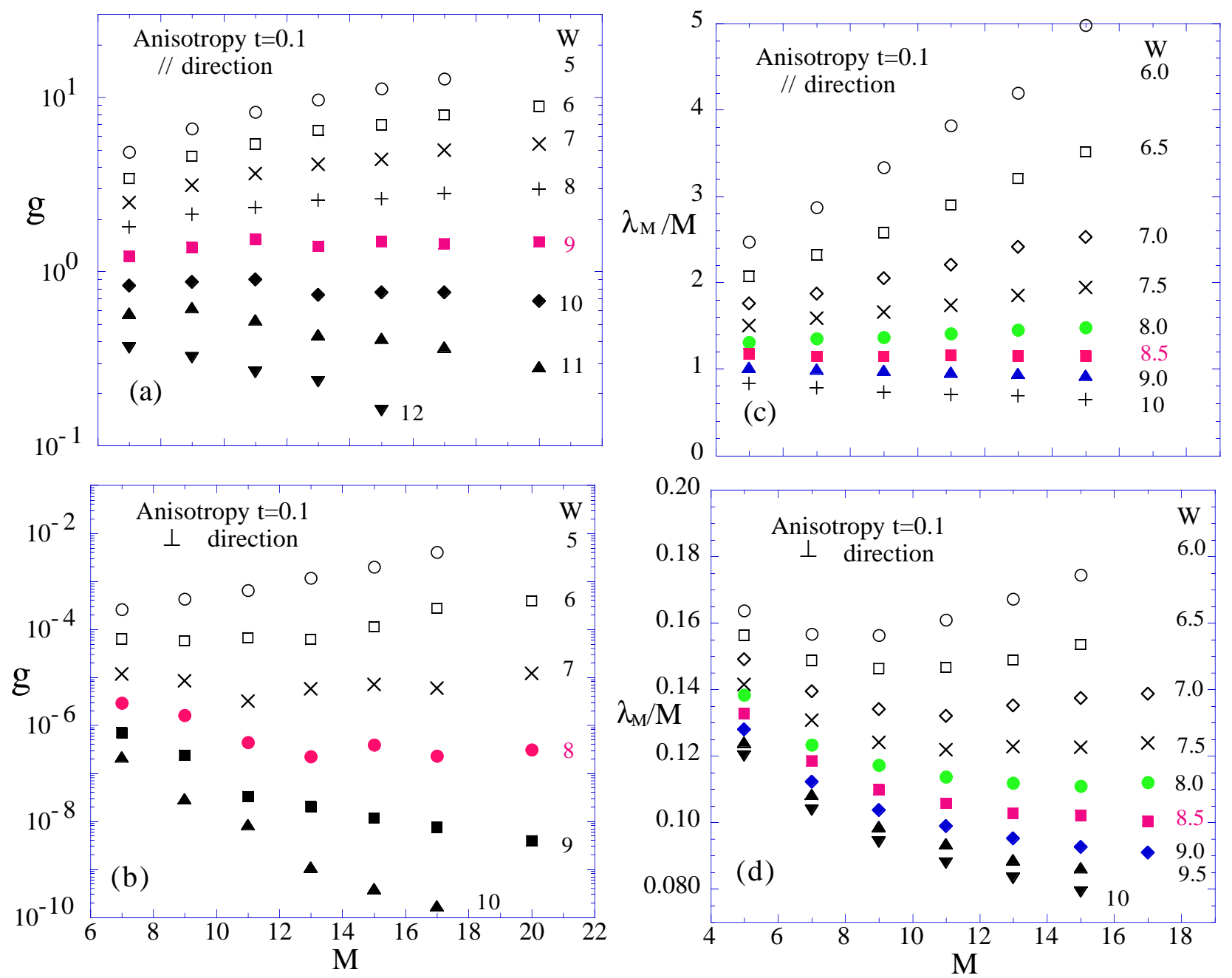
Figure 2

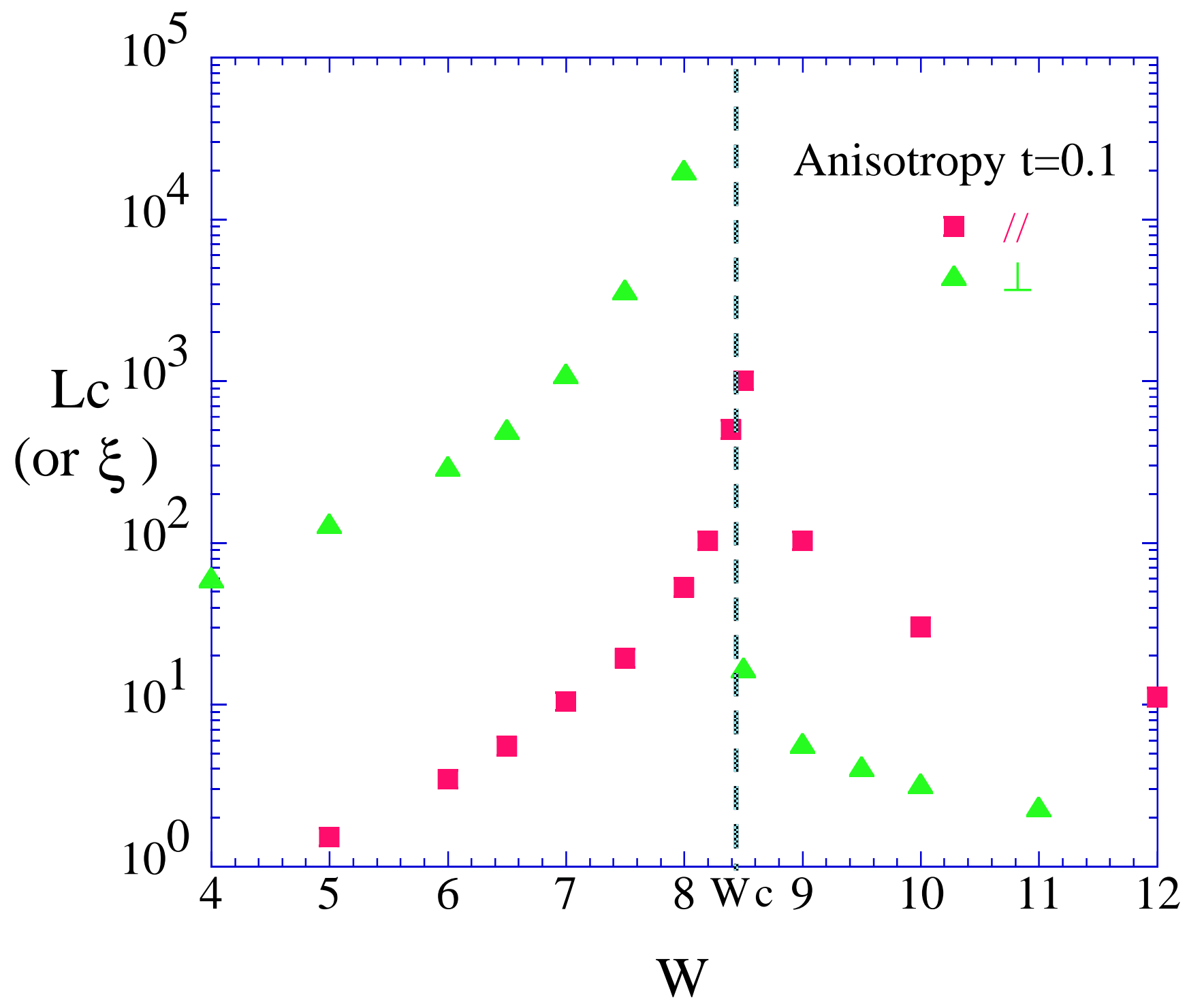


Figure 3

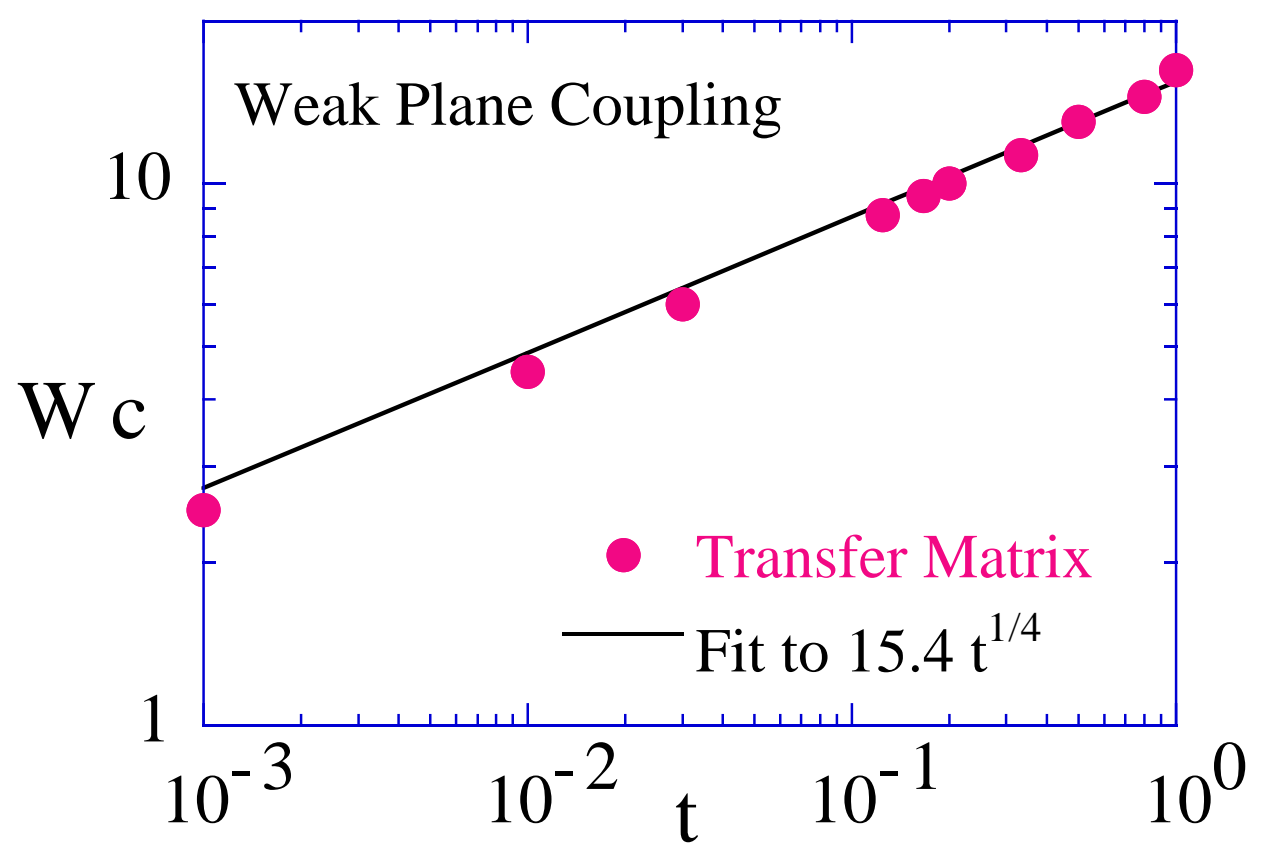

